\title{
Principles of perceptual grouping: implications for image-guided surgery
}

\author{
Birgitta Dresp-Langley* \\ ICube UMR 7357 Centre National de la Recherche Scientifique, University of Strasbourg, Strasbourg, France
}

Keywords: Gestalt theory, law of good continuation, principle of Prägnanz, collinear integration, border ownership, figure-ground, image-guided surgery

The laws and principles which predict how perceptual qualities can be extracted from the most elementary visual signals were discovered by the Gestalt psychologists (e.g., Wertheimer, 1923; Metzger, 1930, translated and re-edited by Spillmann in 2009 and 2012, respectively). Their seminal work has inspired visual science ever since, and has led to exciting discoveries which have confirmed the Gestalt idea that the human brain would have an astonishing capacity for selecting and combining critical visual signals to generate output representations for decision making and action. This capacity of selection and integration enables the perception of form and space, and the correct estimation of relative positions, trajectories, and distances of objects represented in planar images. The Gestalt laws and principles were initially aimed at answering a single all-encompassing question: "Why does the world look the way it does." They have subsequently been made operational in experimental studies (for an illustration of on-going research see the international METHUSALEM project, coordinated by Johan Wagemans, at www.gestaltrevision.be) aimed at deepening our insights into the ways in which specific characteristics and qualities of visual configurations may determine perceptual organization and behavior at various levels of processing. Perceptual organization directly determines the ability of human observers to assess (1) which parts of an image belong together to form a unified visual object or shape, and (2) which parts should be nearer and which further away from the observer if the represented objects were seen in the real world. This opinion paper argues that the Gestalt principle of Prägnanz and the Gestalt law of good continuation address specific problems of perceptual organization with critical implications for visual interface design, and the design of image-guided surgery platforms in particular.

The principle of Prägnanz relates to the general Gestalt postulate that objects in the visual field will produce the simplest and most complete perceptual solution possible under the conditions given. The Gestalt laws of perceptual organization, of which the law of good continuation is a particular example, describe the conditions under which specific perceptual solutions (groupings) are likely to occur. The question of how planar image structures are grouped into perceptual representations of figure and ground is one of the study grounds the Gestalt laws have been designed for. Figure-ground representation is a perceptual solution that enables the observer to assess which objects in the image would be likely to be nearer and which objects would be likely to be further away in a real world configuration. It is mediated by specific image cues to shape and to relative distance, involving local signals of contrast and orientation to fill in specific regions of an image and thereby enabling the perception of surfaces. The associated perceptual sensations of local contrast enhancement make visual objects in the image appear to stand in front of other objects represented in the same plane. Such sensations are often deemed "illusory" because they have no physical origin, i.e., there is no objective difference in local luminance that would explain the resulting percepts (e.g., Heinemann, 1955; Hamada, 1985; O’Shea et al., 1994; De Weert and Spillmann, 1995; Grossberg, 1997; Dresp and Fischer, 2001; Dresp et al., 2002; Guibal and Dresp, 2004; Devinck et al., 2006; Pinna and Reeves, 2006; Dresp-Langley and Reeves, 2012, 2014). An essential aspect of this process of figure-ground segregation is the perceptual assignment of border ownership (see the review 
by von der Heydt on this topic). The Gestalt theorist Rubin (1921) was among the first to point out that a figure has distinct perceptual qualities that make it stand out against the rest of the visual field, which thereby acquires the perceptual quality of ground (or background). A figure occludes the ground and, therefore, owns the borders which separate it from the latter (Craft et al., 2007; Zhang and von der Heydt, 2010). Zhou et al. (2000) found neurons predominantly in V2 (but also V1) of the monkey that respond selectively to the location of borders in the visual field. Selective visual attention to the figure strengthens the neuronal responses to its borders (Qiu et al., 2007).

The Gestalt psychologists also correctly presumed that, to recover a representation of a whole from parts, the brain must achieve the perceptual integration of visual information across collinear space (e.g., Wertheimer, 1923; Metzger, 1930). The visual integration of contrast information across collinear image space plays a crucial role in form vision under conditions of stimulus uncertainty and configurative ambiguity (e.g., Dresp, 1997; Grossberg, 1997). It is governed by the so-called law of good continuation, and reflected by interactive effects between co-axial stimuli in the visual field (Hubel and Wiesel, 1959, 1968; von der Heydt and Peterhans, 1989; Dresp and Bonnet, 1991; Peterhans von der Heydt, 1991; Kapadia et al., 2000; Craft et al., 2007). Specific response activities of visual cortical neurons are triggered by these co-axial interactions (cf. the first observations by Nelson and Frost, 1978; von der Heydt et al., 1984 in monkey visual cortex), revealing the functional properties of brain mechanisms designed to complete physically discontinuous contrast input across collinear visual space. Collinear spatial integration is crucial for the detection of alignment, virtual trajectories, and shape borders in a world where most objects are seen incompletely. It enables a human observer to assess the continuity of image fragments under conditions of diminished visibility and heightened stimulus ambiguity. Experimental data on collinear visual integration have shown that the perceptual recovery of global representations of collinear space involves many levels of visual processing, not a single one, from the visual detection of local image detail to the perception of global association fields (e.g., Dresp, 1993; Field et al., 1993; Polat and Sagi, 1993, 1994; Kapadia et al., 1995; Polat and Norcia, 1996; Yu and Levi, 1997, 2000; Wehrhahn and Dresp, 1998; Chen et al., 2001; Chen and Tyler, 2001; Tzvetanov and Dresp, 2002; Dresp and Langley, 2005; Chen and Tyler, 2008; Huang et al., 2012). In complex images, some visible stimulus fragments appear clearly aligned, others do not. Specific phenomenal conditions of contour relatability (Kellman and Shipley, 1991; Shipley and Kellman, 1992, 2001) need to be satisfied to enable collinear interpolation in static $2 \mathrm{D}$ scenes. This process of interpolation constrains the spreading of surfaces across unspecified regions in the image. The contribution of past experience and perceptual learning to early mechanisms of interpolation and grouping needs to be taken into account given that specific memory data about objects (Kimchi and Hadad, 2002) and their most likely spatial configuration are likely to facilitate (or eventually interfere with, depending on conditions) ongoing visual processing of an image.

Although the recovery of veridical object properties was not a major question in early Gestalt theory, its laws of perceptual organization have generated a conceptual framework for addressing it. Understanding which image conditions produce geometric configurations that will satisfy the most essential laws of Gestalt and ensure optimal Prägnanz for image based decision making is similar to understanding the grammar of well-formed sentences. Gestalt theory is as relevant as ever in the context of visual interface technology for image-guided surgery, for example. Image-guided surgery uses images taken before and/or during the procedure to help the surgeon navigate. The goal is to augment the surgeon's capacity for decision making and action during the procedure (see Perrin et al., 2009, for review). In augmented reality, the guidance is provided directly on the surgeon's view of the patient by mixing real and virtual images (Figure 1). The perceptual qualities (color, brightness,

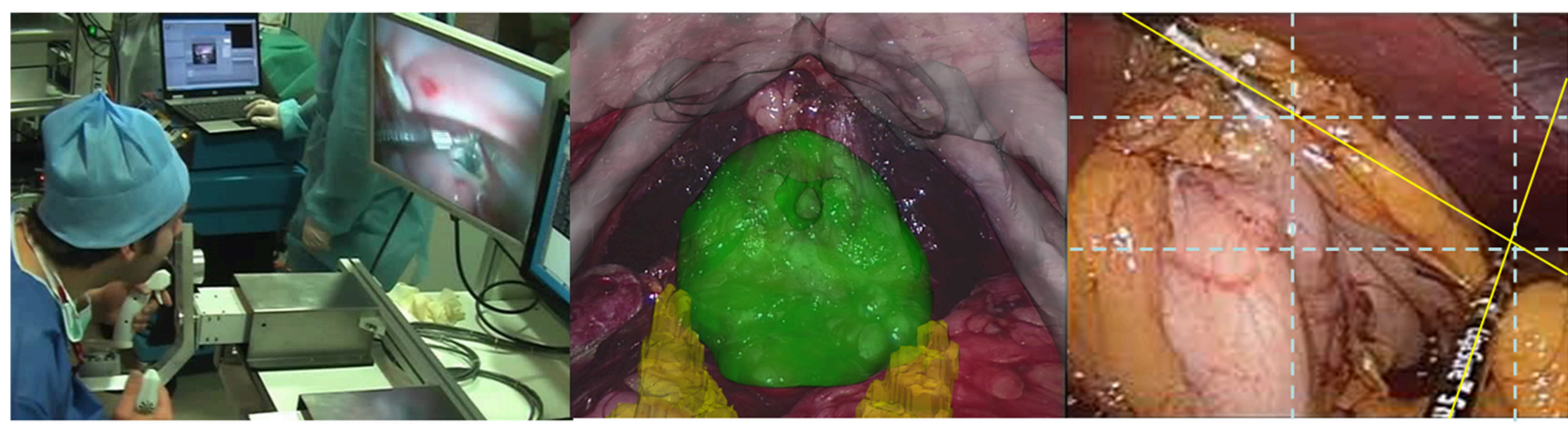

FIGURE 1 | An image configuration will produce the simplest and most complete perceptual solution possible under the conditions given (Gestalt principle of Prägnanz). In image-guided surgery, visual guidance is provided directly on the surgeon's view of the patient's anatomy by mixing real and virtual images. Understanding which image conditions produce geometric configurations that will satisfy the most essential laws of Gestalt and ensure optimal Prägnanz for decision will help increase the efficiency of rendered images (middle). The goal here is to facilitate interventional strategies with regard to specific regions of interest to the surgeon. Visual tracking of the tooltip trajectories is important for evaluating skill evolution, the positional accuracy of the tooltips being critical (left). Technology facilitating the positional accuracy of tool-tip movements by generating visual data for relative position, alignment, and trajectory anticipation (perceptual law of good continuation) is urgently needed. The real-time computational analysis of deviations from critical alignments during interventions (right) is currently the "holy grail" in this field of technological development. 
salience e.a.) of the rendered images are essential for making specific regions of interest to the surgeon optimally perceptible. This includes the visual traceability of devices relative to the patient, the registration and alignment of the preoperative model, and optimized rendering and visualization of the preoperative data. Visualization in this context means translating image data into a graphic representation that is understandable by the user (the surgeon), as it conveys important information for assessing structure and function, and for making (the right!) decisions during an intervention. The field has evolved dramatically in recent years, yet, the most critical problem for image-guided surgery is still the one of task-centered user interface design. During a surgical intervention, the timing of the generation of image data is absolutely critical, and to facilitate navigation through large cavities with multiple potential obstacles, such as within the abdomen, complex displays have been designed to provide navigational aids. They combine surface renderings of anatomy (Figure 1, middle) from preoperative imaging with intra-operative visualization techniques. A common strategy here is representing volumetric data as $2 \mathrm{D}$ surfaces with varying opacity. The efficiency of renderings for facilitating decisions of the human user can be evaluated in terms of the perceptual salience of critical surfaces that represent regions of interest to the surgeon.

\section{REFERENCES}

Chen, C. C., Kasamatsu, T., Polat, U., and Norcia, A. M. (2001). Contrast response characteristics of long-range lateral interactions in cat striate cortex. Neuroreport 12, 655-661. doi: 10.1097/00001756-200103260-00008

Chen, C. C., and Tyler, C. W. (2001). Lateral sensitivity modulation explains the flanker effect in contrast discrimination. Proc. R. Soc. Lond. Ser B 268, 509-516. doi: $10.1098 /$ rspb.2000.1387

Chen, C. C., and Tyler, C. W. (2008). Excitatory and inhibitory interaction fields of flankers revealed by contrast-masking functions. J. Vis. 8, 1-14. doi: $10.1167 / 8.4 .10$

Craft, E., Schüetze, H., Niebur, E., and von der Heydt, R. (2007). A neural model of figure-ground organization. J. Neurophysiol. 97, 4310-4326. doi: 10.1152/jn.00203.2007

Devinck, F., Spillmann, L., and Werner, J. S. (2006). Spatial profile of contours inducing long-range color assimilation. Vis. Neurosci. 23, 573-577. doi: $10.1017 /$ S0952523806233224

De Weert, C. M., and Spillmann, L. (1995). Assimilation: asymmetry between brightness and darkness. Vision Res. 35, 1413-1419. doi: 10.1016/00426989(95)98721-K

Dresp, B. (1993). Bright lines and edges facilitate the detection of small light targets. Spat. Vis. 7, 213-225. doi: 10.1163/156856893X00379

Dresp, B. (1997). On 'illusory' contours and their functional significance. Curr. Psychol. Cogn. 16, 489-517.

Dresp, B., and Bonnet, C. (1991). Psychophysical evidence for low-level processing of illusory contours. Vision Res. 10, 1813-1817. doi: 10.1016/00426989(91)90028-4

Dresp, B., Durand, S., and Grossberg, S. (2002). Depth perception from pairs of overlapping cues in pictorial displays. Spat. Vis. 15, 255-276. doi: $10.1163 / 15685680260174038$

Dresp, B., and Fischer, S. (2001). Asymmetrical contrast effects induced by luminance and color configurations. Percept. Psychophys. 63, 1262-1270. doi: 10.3758/BF03194539

Dresp, B., and Langley, O. K. (2005). Long-range spatial integration across contrast signs: A probabilistic mechanism? Vision Res. 45, 275-284. doi: 10.1016/j.visres.2004.08.018
Moreover, intra-operative imaging often provides further diagnostic information and permits assessing risks as well as perspectives of repair. In this context, image-guided instrument tracking is a major challenge for current research and development in this field (West and Maurer, 2004; Huang et al., 2007). A critical problem for the surgeon is detecting and keeping track of the relative positions of the surgical tools he/she is using during the intervention (Figure 1, right). Visual tracking of the tooltip trajectories is also a precious aid for evaluating skill evolution in trainee surgeons, the positional accuracy of the tooltips being critical during an intervention (e.g., Jiang et al., 2015). The development and testing of new visual aids to facilitate the detection of alignment, relative position and trajectories (perceptual law of good continuation) is urgently needed here. Ultimately, technology where the surgical tool itself will become a genuine visual navigation aid in imageguided surgery is to be developed in the near future and psychophysical testing should have a major impact on these developments.

\section{FUNDING}

Grant support was provided by the Centre National de la Recherche Scientifique (CNRS MI AAP 2015).

Dresp-Langley, B., and Reeves, A. (2012). Simultaneous contrast and apparent depth from true colors on grey: chevreul revisited. Seeing Perceiv. 25, 597-618. doi: 10.1163/18784763-00002401

Dresp-Langley, B., and Reeves, A. (2014). Effects of saturation and contrast polarity on the figure-ground organization of color on gray. Front. Psychol. 5:1136. doi: 10.3389/fpsyg.2014.01136

Field, D. J., Hayes, A., and Hess, R. F. (1993). Contour integration by the human visual system: evidence for a local "association field". Vision Res. 33, 173-193. doi: 10.1016/0042-6989(93)90156-Q

Grossberg, S. (1997). Cortical dynamics of 3-D figure-ground perception of 2-D pictures. Psychol. Rev. 104, 618-658. doi: 10.1037/0033-295X.104. 3.618

Guibal, C. R. C., and Dresp, B. (2004). Interaction of color and geometric cues in depth perception: when does red mean near, Psychol. Res. 10, 167-178. doi: 10.1007/s00426-003-0167-0

Hamada, J. (1985). Asymmetric lightness cancellation in Craik-O'Brien patterns of negative and positive contrast. Biol. Cybern. 52, 117-122. doi: 10.1007/BF00364002

Heinemann, E. G. (1955). Simultaneous brightness induction as a function of inducing and test-field luminance. J. Exp. Psychol. 50, 89-96. doi: 10.1037/h0040919

Huang, J., Triedman, J. K., Vasilyev, N. V., Suematsu, Y., Cleveland, R. O., and Dupont, P. E. (2007). Imaging artefacts of medical instruments in ultrasoundguided interventions. J. Ultrasound Med. 26, 1303-1322.

Huang, P. C., Chen, C. C., and Tyler, C. W. (2012). Collinear facilitation over space and depth. J. Vis. 12, 1-9. doi: 10.1167/12.2.20

Hubel, D. H., and Wiesel, T. N. (1959). Receptive fields of single neurons in the cat's striate cortex. J. Physiol. 148, 574-591. doi: 10.1113/jphysiol.1959.sp006308

Hubel, D. H., and Wiesel, T. N. (1968). Receptive fields and functional architecture of monkey striate cortex. J. Physiol. 195, 215-243. doi: 10.1113/jphysiol.1968.sp008455

Jiang, X., Zheng, B., and Atkins, M. S. (2015). Video processing to locate the tooltip position in surgical eye-hand coordination tasks. Surg. Innovat. 22, 285-293. doi: 10.1177/1553350614541859

Kapadia, M. K., Ito, M., Gilbert, C. D., and Westheimer, G. (1995). Improvement in visual sensitivity by changes in local context: parallel studies in human 
observers and in V1 of alert monkeys. Neuron 15, 843-856. doi: 10.1016/08966273(95)90175-2

Kapadia, M. K., Westheimer, G., and Gilbert, C. D. (2000). Spatial contribution of contextual interactions in primary visual cortex and in visual perception. J. Neurophysiol. 84, 2048-2062.

Kellman, P. J., and Shipley, T. F. (1991). A theory of visual interpolation in object perception. Cogn. Psychol. 23, 141-221. doi: 10.1016/0010-0285(91)90009-D

Kimchi, R., and Hadad, B. S. (2002). Influence of past experience on perceptual grouping. Psychol. Sci. 13, 41-47. doi: 10.1111/1467-9280.00407

Metzger, W. (1930). Gesetze des Sehens, English trans. L. Spillmann (2009) Laws of Seeing. Cambridge, MA: MIT Press.

Nelson, J. I., and Frost, B. J. (1978). Orientation-selective inhibition from beyond the classic receptieve field. Brain Res. 139, 359-365.

O'Shea, R. P., Blackburn, S. G., and Ono, H. (1994). Contrast as a depth cue. Vision Res. 34, 1595-1604.

Perrin, D. P., Vasilyev, N. V., Novotny, P., Stoll, J., Howe, R. D., Dupont, P. E., et al. (2009). Image guided surgical interventions. Curr. Probl. Surg. 46, 730-766. doi: 10.1067/j.cpsurg.2009.04.001

Peterhans, E., and von der Heydt, R. (1991). Subjective contours-bridging the gap between psychophysics and physiology. Trends Neurosci. 14, 112-119. doi: 10.1016/0166-2236(91)90072-3

Pinna, B., and Reeves, A. (2006). Lighting, backlighting, and the laws of figurality in the watercolor illusion. Spat. Vis. 19, 341-373. doi: $10.1163 / 156856806776923434$

Polat, U., and Norcia, A. M. (1996). Neurophysiological evidence for contrast dependent long-range facilitation and suppression in human visual cortex. Vision Res. 36, 2099-2109. doi: 10.1016/0042-6989(95)00281-2

Polat, U., and Sagi, D. (1993). Lateral interactions between spatial channels: suppression and facilitation revealed by lateral masking experiments. Vision Res. 33, 993-999. doi: 10.1016/0042-6989(93)90081-7

Polat, U., and Sagi, D. (1994). The architecture of perceptual spatial interaction. Vision Res. 34, 73-78. doi: 10.1016/0042-6989(94)90258-5

Qiu, F. T., Sugihara, T., and von der Heydt, R. (2007). Figure-ground mechanisms provide structure for selective attention. Nat. Neurosci. 10, 1492-1499. doi: $10.1038 / \mathrm{nn} 1989$

Rubin, E. (1921). Visuell Wahrgenommene Figuren: Studien in psychologischer Analyse. Kopenhagen: Gyldendalske.

Shipley, T. F., and Kellman, P. J. (1992). Strength of visual interpolation depends on the ratio of physically specified to total edge length. Percept. Psychophys. 52, 97-106. doi: 10.3758/BF03206762
Shipley, T. F., and Kellman, P. J. (eds.). (2001). From Fragments to Objects: Segmentation and Grouping in Vision. Amsterdam: Elsevier Science Press.

Tzvetanov, T., and Dresp, B. (2002). Short- and long-range effects in line contrast detection. Vision Res. 42, 2493-2498. doi: 10.1016/S0042-6989(02)00198-0

von der Heydt, R., and Peterhans, E. (1989). Mechanisms of contour perception in monkey visual cortex: I. Lines of pattern discontinuity. J. Neurosci. 9, 1731-1748.

von der Heydt, R., Peterhans, E., and Baumgartner, G. (1984). Illusory contours and cortical neuron responses. Science 224, 1260-1262. doi: 10.1126/science.6539501

Wehrhahn, C., and Dresp, B. (1998). Detection facilitation by collinear stimuli in humans: dependence on strength and sign of contrast. Vision Res. 38, 423-428. doi: 10.1016/S0042-6989(97)00141-7

Wertheimer, M. (1923). Perceived Motion and Figural Organization, English trans. L. Spillmann, M. Wertheimer, K. W. Watkins, S. Lehar, and V. Sarris (2012). Cambridge, MA: MIT Press.

West, J. B., and Maurer, C. R. Jr. (2004). Designing optically tracked instruments for image-guided surgery. IEEE Trans. Med. Imaging 23, 533-545. doi: 10.1109/TMI.2004.825614

Yu, C., and Levi, D. M. (1997). Spatial facilitation predicted with end-stopped spatial filters. Vision Res. 37, 3117-3128. doi: 10.1016/S0042-6989(97)00120-X

Yu, C., and Levi, D. M. (2000). Surround modulation in human vision unmasked by masking experiments. Nat. Neurosci. 3, 724-728. doi: 10.1038/ 76687

Zhang, N. R., and von der Heydt, R. (2010). Analysis of the context integration mechanisms underlying figure-ground organization in the visual cortex. J. Neurosci. 30, 6482-6496. doi: 10.1523/JNEUROSCI.5168-09.2010

Zhou, H., Friedman, H. S., and von der Heydt, R. (2000). Coding of border ownership in monkey visual cortex. J. Neurosci. 20, 6594-6611.

Conflict of Interest Statement: The author declares that the research was conducted in the absence of any commercial or financial relationships that could be construed as a potential conflict of interest.

Copyright $\odot 2015$ Dresp-Langley. This is an open-access article distributed under the terms of the Creative Commons Attribution License (CC BY). The use, distribution or reproduction in other forums is permitted, provided the original author(s) or licensor are credited and that the original publication in this journal is cited, in accordance with accepted academic practice. No use, distribution or reproduction is permitted which does not comply with these terms. 